\title{
A survey on digital forensics trends
}

\begin{abstract}
Digital forensic has evolved from addressing minor computer crimes to investigation of complex international cases with massive effect on the world. This paper studies the evolution of the digital forensic; its origins, its current position and its future directions. This paper sets the scene with exploring past literature on digital forensic approaches followed by the assessment and analysis of current state of art in both industrial and academic digital forensics research. The obtained results are compared and analyzed to provide a comprehensive view of the current digital forensics landscape. Furthermore, this paper highlights critical digital forensic issues that are being overlooked and not being addressed as deserved. The paper finally concludes with offering future research directions in this area.
\end{abstract}

Keyword: Digital forensic; Mobile device forensic; Forensic framework; Network forensic; String analysis 